\title{
Nattokinase Crude Extract Inhibits Hepatocellular Carcinoma Growth in Mice
}

\author{
Yongmin Yan, Yanjing Wang, Jiali Qian, Sihui Wu, Yi Ji, Yanxiao Liu, Jian Zeng, and Aihua Gong* \\ Key Laboratory of Laboratory Medicine of Jiangsu Province, School of Medicine, Jiangsu University, Zhenjiang, 212013, Jiangsu, P.R. China
}

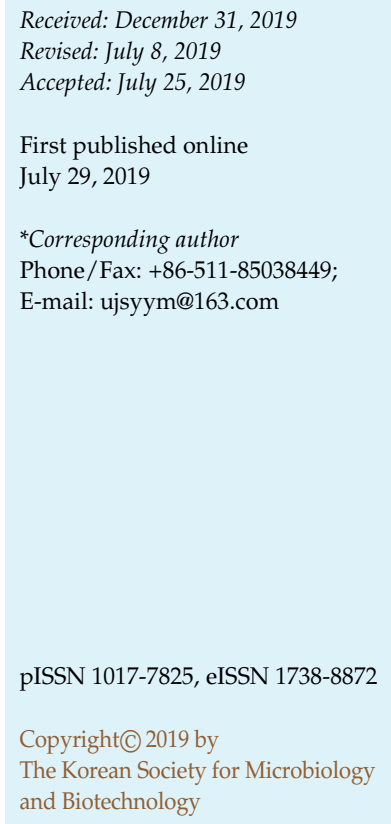

Nattokinase (NK, E.C. 3.4.21.62) is a serine protease produced by Bacillus subtilis natto that shows promise for the treatment of thrombotic disease. In this study, we assessed the effects of NK on the development of hepatocellular carcinoma (HCC), a principal malignancy of the liver that causes morbidity and mortality worldwide. Crude extracts of NK (NCE) were isolated from fermentation medium by centrifugation and separated into three fractions $(<10$ $\mathrm{K}, 100 \sim 30 \mathrm{~K}$ and $>30 \mathrm{~K}$ ). Orthotopic HCC mouse models were established and NCE was administered by oral gavage. H\&E staining was performed to examine the pathology of HCC livers. Immunohistochemistry and immunofluorescence were used to evaluate FOXM1, CD31, CD44 and vimentin expression in the liver. Compared to PBS groups, NCE increased the survival rates of HCC-bearing mice to $31 \%$ and decreased ascites. Low-intensity ultrasound imaging showed that the hypoechoic mass area was lower in NCE-treated mice and that tumor growth significantly decreased. IHC staining showed that the expression of FOXM1 was inhibited by NCE treatment. Immunofluorescence results revealed lower levels of CD31, CD44 and vimentin in the NCE groups. Taken together, these data demonstrate that NCE from Bacillus subtilis natto improves survival and inhibits tumor growth in HCC mice.

Keywords: Hepatocellular carcinoma, nattokinase, tumor growth, cancer

\section{Introduction}

Hepatocellular carcinoma (HCC) is one of the most common neoplasms and a leading cause of cancer-related death [1]. The incidence of HCC continues to rise due to the prevalence of hepatitis B and hepatitis C infection [2]. Resection (LR), liver transplantation (LT) and radiofrequency ablation are curative treatment options [3]. However, metastasis or recurrence are frequent leading to poor prognosis [4]. New complementary anti-HCC therapies are thus urgently required.

Nattokinase (NK, E.C. 3.4.21.62) is a serine protease composed of 275 amino acids that is produced by Bacillus subtilis natto, a traditional soybean food of B. subtilis natto fermentation [5]. NK has reported fibrinolytic activity [6] and is used in the treatment of thrombotic disease due to its safety, low-cost, efficiency and stability [7]. NK-degradation products reduce hypertension by preventing elevated plasma angiotensin II levels [8]. The consumption of NK in the North American hypertensive population is associated with beneficial changes in blood pressure. Randomized and controlled clinical trials also show that the increased intake of NK can prevent and treat hypertension [9]. Other applications of NK include the treatment of cerebral ischemia, hypertension, Alzheimer's disease and vitreoretinal disorders. For example, intravitreal injection of NK alleviates pharmacological vitreolysis due to the induction of posterior vitreous detachment (PVD) [10]. The oral administration of NK has therapeutic applications for the treatment of AD through modulating brain acetylcholinesterase (AchE) activity, TGF- $\beta$, Fas and IL-6 levels [11]. The effects of $\mathrm{NK}$ in cancer prevention have not been investigated to date.

In this study, NK was produced from B. subtilis natto and its ability to prevent HCC in mouse models was investigated. Nattokinase crude extracts (NCE) were isolated from the fermentation medium of cultured B. subtilis natto by centrifugation. We found that oral administration of 
NCE significantly improved the survival of HCC mice and decreased tumor growth. Furthermore, NCE treatment led to a reduction of FOXM1, CD31 and vimentin expression. FOXM1 and CD31 are neovascularization markers whilst CD44 and vimentin are proliferation-associated factors essential for HCC tumorigenesis [12-15]. This highlights the benefits of NCE for HCC treatment.

\section{Materials and Methods}

\section{Medium and Culture Conditions}

NCE was produced as previously described [16]. Briefly, $B$. subtilis natto was maintained in Luria-Bertani (LB) medium (1 $\mathrm{g}$ of sodium chloride, $1 \mathrm{~g}$ of peptone, $0.5 \mathrm{~g}$ of yeast extract and $1.5 \mathrm{~g}$ of agar in $100 \mathrm{ml}$ of deionized water) $\left(\mathrm{pH} \mathrm{7.0)}\right.$ at $37^{\circ} \mathrm{C}$. Seed cultures were inoculated $(0.5 \%, \mathrm{v} / \mathrm{v})$ into fermentation medium for NK production. The fermentation medium was comprised of $50 \mathrm{~g}$ of red bean powder, $20 \mathrm{~g}$ of glucose, $5 \mathrm{~g}$ of sodium chloride, $1 \mathrm{~g}$ of dipotassium hydrogen phosphate, $1 \mathrm{~g}$ of potassium dihydrogen phosphate, $0.1 \mathrm{~g}$ of calcium chloride and $0.5 \mathrm{~g}$ of magnesium sulfate. Cells were grown in $500 \mathrm{ml}$ flasks containing $100 \mathrm{ml}$ of medium and were incubated in a shaker at $225 \mathrm{rpm}$ at $37^{\circ} \mathrm{C}$. The human HCC cell line Hepa1-6 (H-2b) was purchased from Boster Biological Technology Ltd. (Wuhan, China) and cultured in DMEM containing $2 \mathrm{mM}$ of glutamine and $10 \%$ FBS as per the manufacturer's instructions.

\section{Isolation of NCE}

NCE supernatants were extracted from the fermentation medium by centrifugation for $8 \mathrm{~min}$ at $12,000 \times g$. Supernatants were separated with $10 \mathrm{~K}$ and $30 \mathrm{~K}$ ultrafiltration membranes (Millipore, Ireland) according to molecular weight. NCE fractions were divided into $<10 \mathrm{~K}, 10 \sim 30 \mathrm{~K}$, and $>30 \mathrm{~K}$. The molecular weight of NK is $\sim 28 \mathrm{~K}$ and $10 \sim 30 \mathrm{~K}$ fractions were defined as NK extracts.

\section{Fibrinolytic Activity of NCE}

The fibrinolytic activity of NCE was measured using the fibrin degradation method. Briefly, fibrin-substrate solution was mixed
A

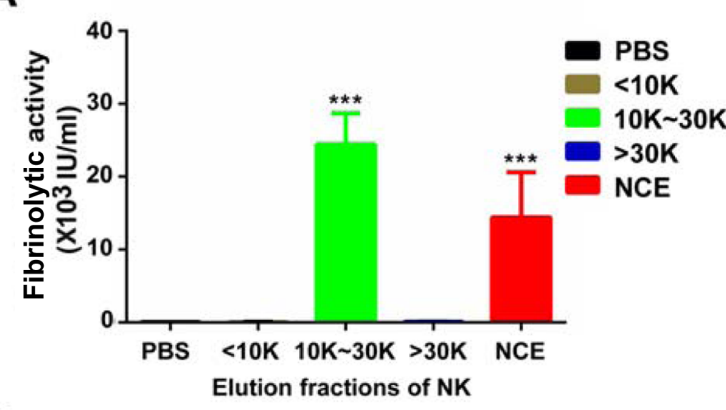

C

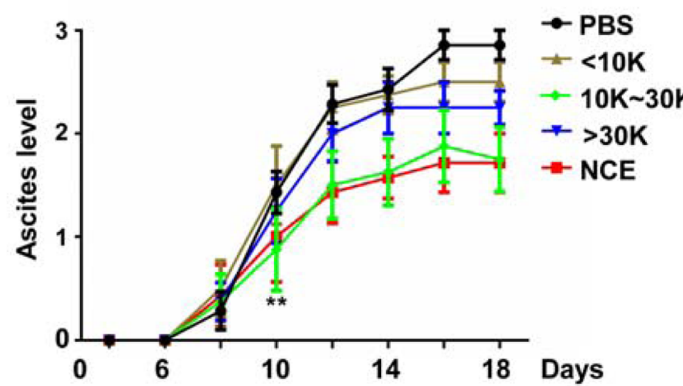

E

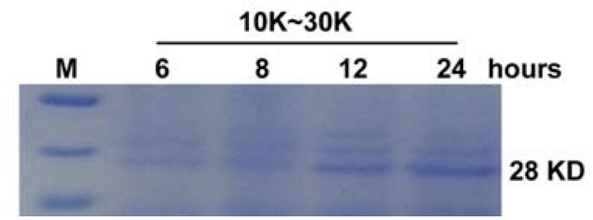

B

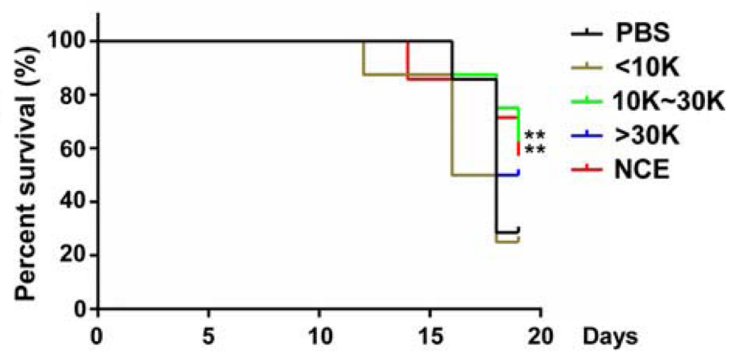

D

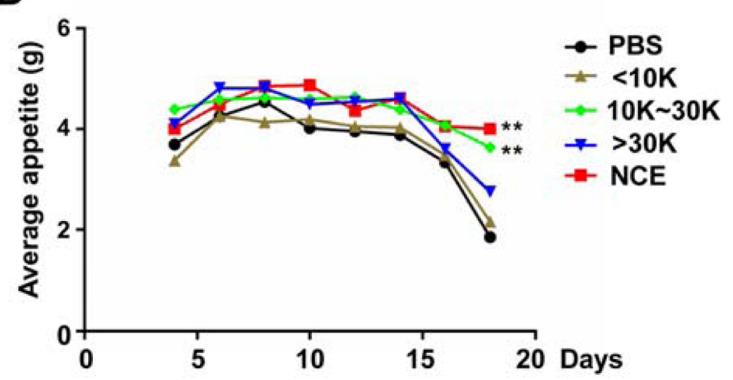

Fig. 1. Nattokinase enhanced survival of HCC-bearing mice.

(A) Fibrinolytic activity of NCE with different molecular weight components from the culture supernatants of B. subtilis $(n=5$, *** $p<0.001)$. (B) Survival of HCC-bearing mice on each day $\left({ }^{* *} p<0.01\right)$. (C) Ascites levels of HCC-bearing mice on each day $\left(n=8,{ }^{* *} p<0.01\right)$. (D). Average appetite of HCC-bearing mice on each day $\left(n=8,{ }^{* *} p<0.01\right)$. $<10 \mathrm{~K}, 10 \sim 30 \mathrm{~K}$, and $>30 \mathrm{~K}$ : fractions of NCE with different molecular weights. (E) SDS-PAGE analysis of 10 30 K fractions of NCE from Bacillus subtilis natto inoculated fermentation medium at 6, 8, 12, and $24 \mathrm{~h}$. 
with $0.1 \mathrm{ml} \mathrm{NK}$ fractions and incubated at $37^{\circ} \mathrm{C}$ for $60 \mathrm{~min}$. PBS and unseparated NCE were used as controls. Trichloroacetic acid (TCA, $2 \mathrm{ml}$ ) solution was added to stop the reaction. Samples were centrifuged at 13,000 $\times g$ for $10 \mathrm{~min}$ and the absorbance at $275 \mathrm{~nm}$ was measured. The fibrinolytic activity (FU) of NCE was defined as the amount of enzyme leading to a 0.01 increase in absorbance in $1 \mathrm{~min}$.

\section{Establishment of Orthotopic HCC Mouse Models and NCE Treatment}

All the animal experiments were authorized and approved by the Ethics Committee of Jiangsu University (2012258). C57BL/6 wild-type mice (6-8 weeks old and weighing $20 \pm 2 \mathrm{~g}$ ) were purchased from the Animal Centre of Jiangsu University (China). HCC models in situ were established through the intrahepatic inoculation of HCC cells. HCC cells $\left(1 \times 10^{5}\right)$ were suspended in $5 \mu \mathrm{l}$ of PBS and injected into the liver with a syringe at an angle of $30^{\circ}$. To avoid bleeding and tumor cell leakage from the injection site, the liver surface was covered with Gelfoam for $5 \mathrm{~min}$ and the peritoneum and skin were closed. Subsequently, HCC mouse models were established. Different fractions of NCE $(<10 \mathrm{~K}$, $10 \sim 30 \mathrm{~K}$, and $>30 \mathrm{~K}$ ) and unseparated NCE were administered by oral gavage at a dose of $7.5 \mu \mathrm{l} / \mathrm{g}$ according to body weight. Mice were randomized into five groups: PBS $(n=16),<10 \mathrm{~K}(n=16)$, $10 \sim 30 \mathrm{~K}(n=16),>30 \mathrm{~K}(n=16)$, and unseparated NCE $(n=14)$ by oral gavage. Survival of the mice was assessed after 20 days.

\section{Hematoxylin and Eosin (H\&E) Staining}

$\mathrm{H} \& \mathrm{E}$ staining was used to examine the pathology of the livers from HCC mouse models. Briefly, liver tissues were fixed with $4 \%$ formaldehyde and sections were embedded in paraffin $(4 \mu \mathrm{m})$. Sections were stained with hematoxylin and eosin according to standard protocols and analyzed by microscopy.

\section{Immunohistochemistry and Immunofluorescence}

Following deparaffination and rehydration, liver sections were steamed in citrate buffer (10 mM, pH 6.0) for $30 \mathrm{~min}$ for antigen retrieval and exposed to $3 \%$ hydrogen peroxide for $30 \mathrm{~min}$ to inhibit endogenous peroxidase activity. Slides were blocked in 5\% BSA for $1 \mathrm{~h}$ and incubated with primary antibodies against FOXM1 (USA), CD31 (Bioworld, USA), CD44 (Bioworld) and vimentin (Bioworld) overnight. For immunohistochemistry assays of FOXM1, slides were incubated in secondary antibodies for $30 \mathrm{~min}$ at $37^{\circ} \mathrm{C}$ and visualized with $3,3^{\prime}$-diaminobenzidine followed by counterstaining with hematoxylin (Nikon Eclipse TiS, Japan). Sections were imaged at $200 \times$ magnification. For immunofluorescence assays, slides were labeled with CD31 and stained with Cy3-labeled anti-rabbit IgG secondary antibodies $(1: 800)$ at $37^{\circ} \mathrm{C}$ for $45 \mathrm{~min}$. Nuclei were counterstained with Hoechst 33342 (1:200; Sigma-Aldrich). Images were acquired sequentially on a fluorescent microscope at $200 \times$ (Nikon Eclipse Ti-S).

\section{SDS-PAGE}

Fractions $(10 \sim 30 \mathrm{~K})$ of NCE were collected from B. subtilis natto inoculated fermentation medium at $6,8,12$, and $24 \mathrm{~h}$ respectively. The concentration of collected $10 \sim 30 \mathrm{~K}$ fractions was detected by Bradford assay. Proteins $(10-30 \mu \mathrm{g})$ were separated on $10 \%$ SDSPAGE gels. For the analysis of NK expression, coomassie brilliant blue-stained PAGE gels were scanned using the Synaeme Bioimaging System.

\section{Statistical Analysis}

Data are expressed as the mean \pm SEM. Statistical differences were analyzed by a Student's t-test (two-tailed) with Prism software (GraphPad, USA). Fibrinolytic activity and tumor volume assessments were repeated five times. The ascites levels and average appetite were examined in 8 mice. P-values $<0.05$ were considered significant

\section{Results}

\section{NCE Enhances the Survival of Hepatocellular Carcinoma (HCC) in Mice}

NK activity was detected in the supernatants of B. subtilis. The results showed that NK activity was undetectable in
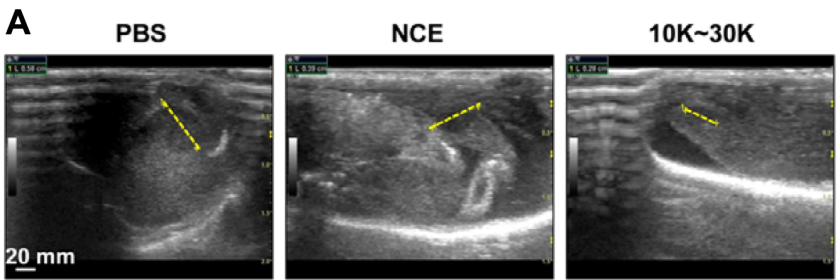

B
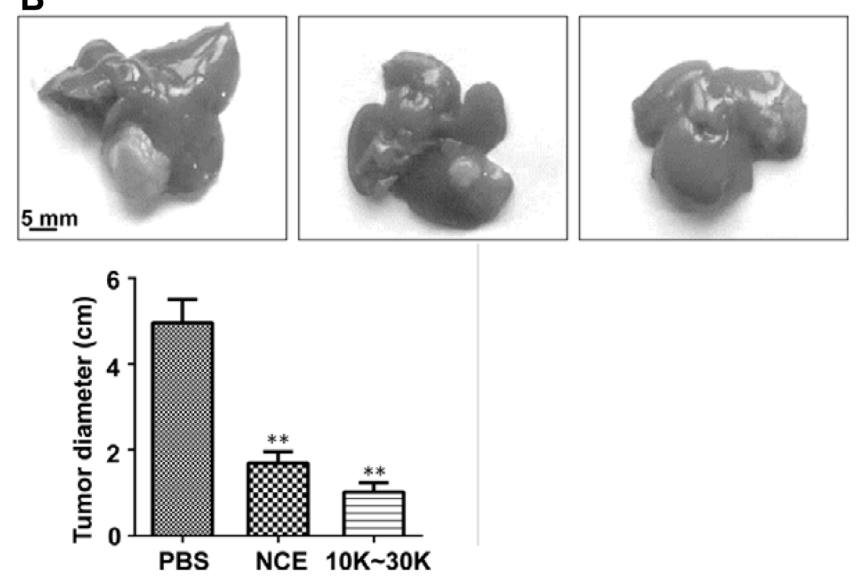

Fig. 2. Nattokinase suppresses tumor growth in HCC-bearing mice.

(A) Low-intensity ultrasound imaging of the tumor area in HCCbearing mice treated with PBS, NCE and $10 \mathrm{~K} \sim 30 \mathrm{~K}$ fractions at 20 days. (B) Tumor nodules on the surface of the liver from HCCbearing mice treated with PBS, NCE and $10 \mathrm{~K} \sim 30 \mathrm{~K}$ fractions at 20 days $\left(n=5,{ }^{* *} p<0.01\right)$. 
PBS, $<10 \mathrm{~K}$ and $>30 \mathrm{~K}$ fractions. NCE from the B. subtilis culture media had enzyme activity of $\sim 14,500 \mathrm{u} / \mathrm{ml}$, which increased to $24,300 \mathrm{u} / \mathrm{ml}$ in $10 \sim 30 \mathrm{~K}$ fractions (Fig. 1A). To assess the therapeutic potential of NK in HCC, NCE was administered to carcinoma in situ models for HCC in C57BL/6J mice by oral gavage. In response to the administration of $10 \sim 30 \mathrm{~K}$ fractions and NCE, $62.5 \%$ and $57.14 \%$ of HCC mice survived respectively. However, the survival rates decreased to $25 \%, 25 \%$ and $31 \%$ in PBS, $<10 \mathrm{~K}$ fraction and $>30 \mathrm{~K}$ fractions, respectively (Fig. 1B). Compared to PBS groups and $<10 \mathrm{~K}$ and $>30 \mathrm{~K}$ groups (Fig. 1C), the ascites levels of HCC-bearing mice decreased in 10 30 K fractions and the NCE groups. The average appetite of HCC-bearing mice consistently increased in $10 \sim 30 \mathrm{~K}$ fractions and the NCE group compared to PBS and $<10 \mathrm{~K}$ and $>30 \mathrm{~K}$ fractions (Fig. 1D). SDS-PAGE also showed that NK of $28 \mathrm{KDa}$ was expressed in 10 30 K fractions, the expression of which increased with fermentation time from 6 to $24 \mathrm{~h}$ (Fig. 1E). These results revealed that NCE enhances the survival of HCC-bearing mice with hepatic carcinoma in situ.

\section{NK Suppresses Tumor Growth in HCC-Bearing Mice}

To investigate the role of NK in liver tumor growth, lowintensity ultrasound imaging was used to examine the tumor area of HCC in situ. Ultrasounds showed that the hypoechoic mass area of liver cancer was inhibited for 15 days after 10 30 K fractions and NCE treatment (Fig. 2A). Tumor nodules visible on the surface of the liver were measured after 20 days of treatment with 10 30 K fractions and NCE treatment (Fig. 2B). The results showed that tumor diameter significantly decreased in 10 30 K fractions and the NCE group. These findings indicated that NCE can suppress in situ tumor growth.

\section{NCE Inhibits HCC Cell Proliferation In Vivo}

To further investigate the components of NCE that inhibit liver tumor growth, H\&E staining was used to examine the pathological changes of NCE-treated liver tumor tissue. In comparison to PBS control groups, the tumor cell area was inhibited and hepatocyte growth was enhanced in the liver tissues from 10 30 K-fractions and NCE-treated mice (Fig. 3A). Forkhead box M1 (FOXM1),
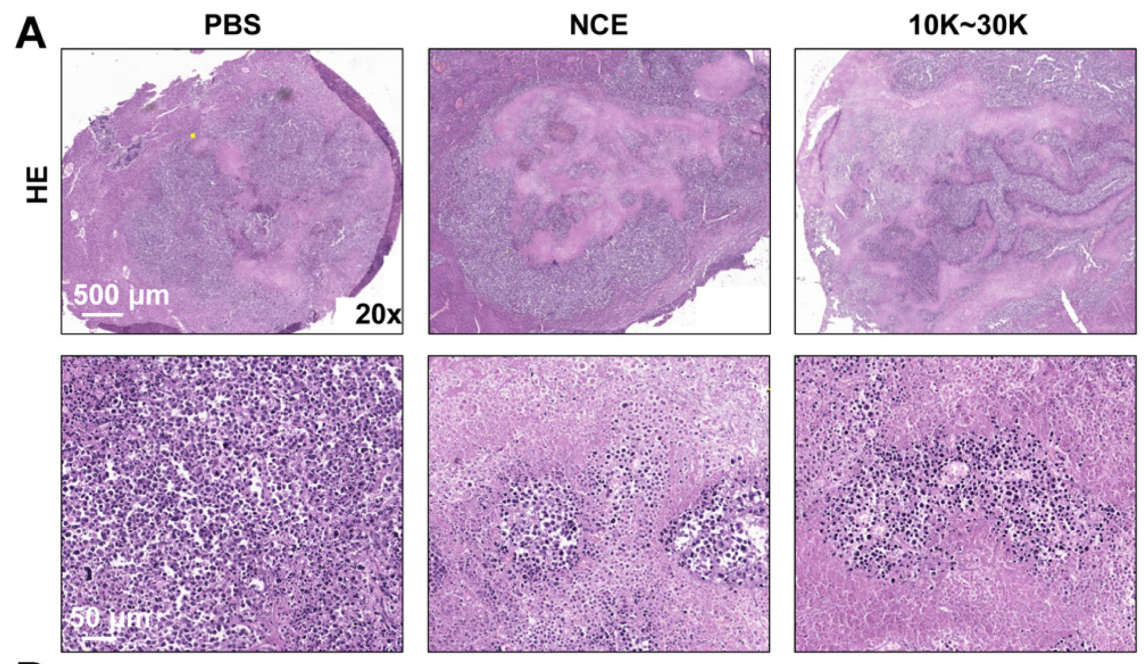

B
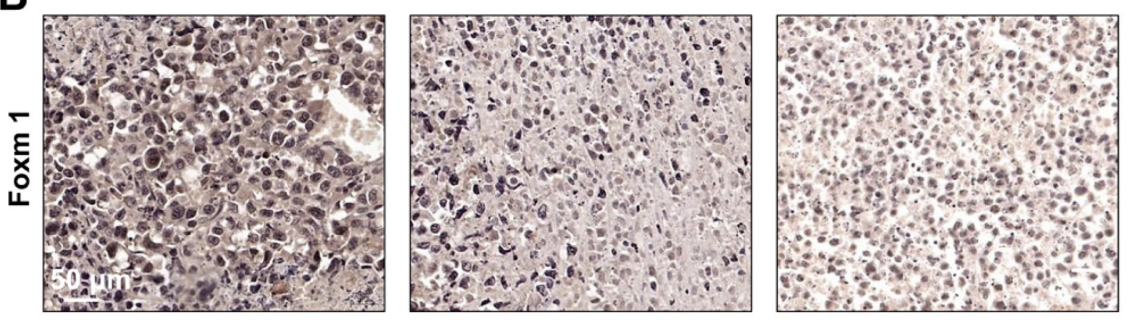

Fig. 3. Immunohistochemistry of FOXM1 in liver tumors.

(A) H\&E staining of tumor tissues from HCC-bearing mice treated with PBS, NCE and 10K 30K fractions at 20 days $(200 \times$, scale bar =). (B) Expression of FOXM1 in tumor tissue from HCC-bearing mice treated with PBS, NCE and 10K 30K fractions at 20 days were investigated by immunohistochemistry $(200 \times)$. 

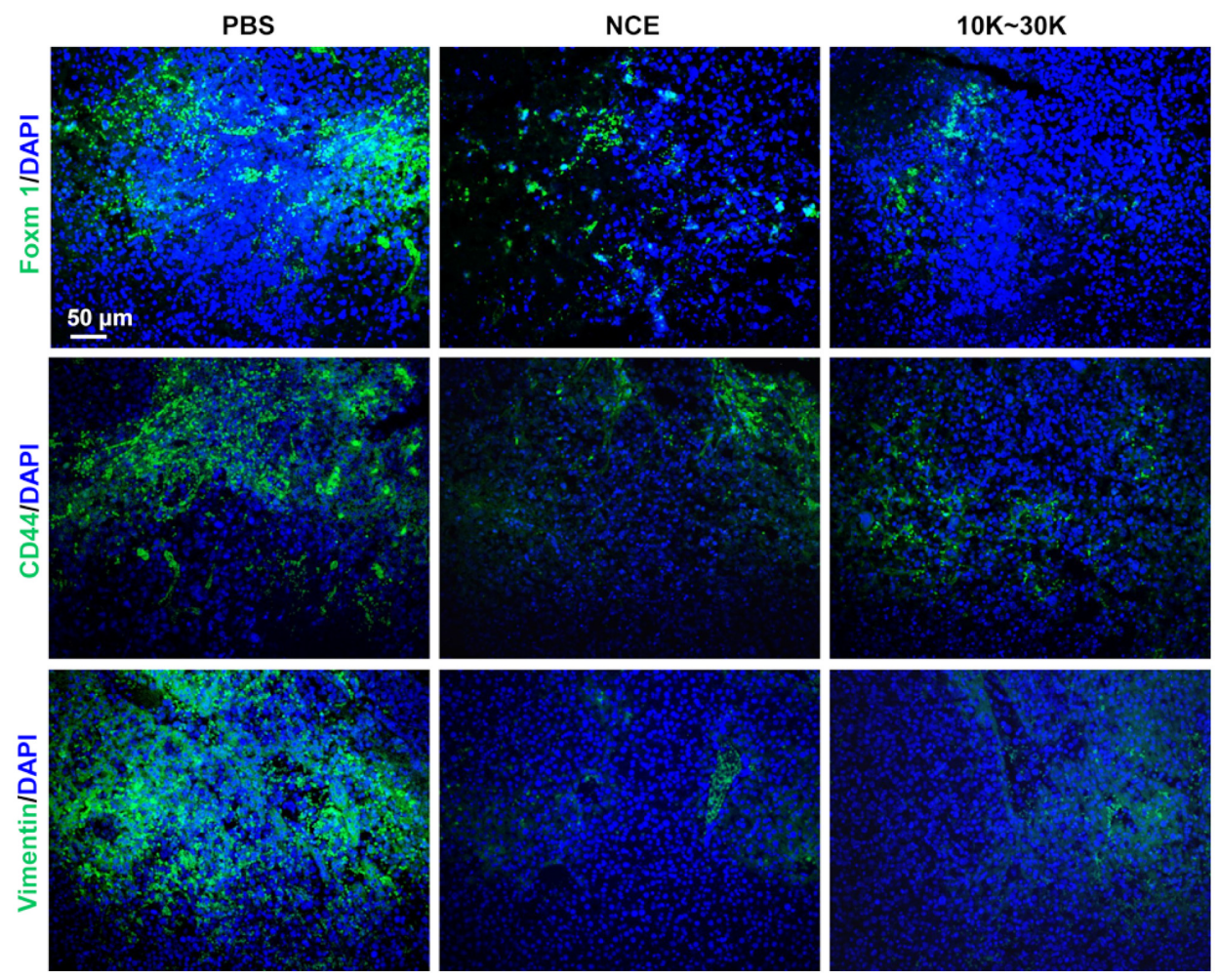

Fig. 4. Immunofluorescence staining of CD31, CD44 and vimentin in liver tumors.

Expression of CD31, CD44 and vimentin in tumor tissue from HCC-bearing mice treated with PBS, NCE and 10K 30K fractions at 20 days were investigated by immunofluorescence $(200 \times)$.

CD44 and vimentin are considered principal regulators of cell differentiation and proliferation and are overexpressed in liver cancer [12-14]. CD31 is an auxiliary neovascularization marker. IHC staining revealed decreased expression of FOXM1 in liver tumor samples from 10 30 Kfractions and NCE-treated mice (Fig. 3B). Immunofluorescence staining showed that CD31, CD44 and vimentin expression were reduced in liver tumor samples from 10 30 K-fractions and NCE-treated mice (Fig. 4). These results demonstrate that NCE suppresses tumor growth through inhibiting cell proliferation in the tumors.

\section{Discussion}

NK was extracted from traditional fermented food using B. subtilis natto (Sumi et al. 1987) [17]. The effects of NK on thrombosis have been confirmed in previous studies. This study investigated the efficacy of NK in hepatic cancer prevention in a mouse models. Amongst the components of B. subtilis culture medium, only $10 \sim 30 \mathrm{~K}$ and NCE exhibited NK activity. The molecular weight of $\mathrm{NK}$ is $28 \mathrm{Kda}$. Thus, it can be inferred that NK is present in the
$10 \sim 30 \mathrm{~K}$ fractions and represents the NCE component of B. subtilis supernatants. In comparison to a placebo, beneficial changes were found for survival and tumor growth inhibition in NK-treated mice. In this study, improved survival rates in mice with HCC were observed in 10 30 K and NCE groups. The inhibition of ascites levels and improved appetites were found in 10 30 K and NCE groups. The survival status of mice with HCC was associated with tumor growth.

Tumor growth is a complex physiological process involving interactions between cells, growth factors, cancer stem cells and cell cycle regulators [18-20]. Cancer therapy studies have highlighted the importance of vascular and vessel proliferation in tumor tissue [21-23]. The supplementation of dietary natto extracts can suppress intimal thickening in response to endothelial injury in rat femoral arteries [24]. Natto extracts suppress intimal thickening after vascular injury as a result of the inhibition of mural thrombi formation. This suggests that NK plays a role in angiogenesis. The results of this study indicated that NK suppresses the expression of the crucial neovascularization markers CD31, FOXM1, CD44 and vimentin [12-15]. These 
transcription factors regulate the proliferation, survival and drug resistance of various cancers [25]. Current cancer therapy focuses on new targets for cancer genesis. The depletion of FOXM1 promotes the anti-tumor activity of PF in colorectal cancer cells [26]. Inhibition of FOXM1 in human PIMAs inhibited mucinous characteristics and reduced tumor growth and invasion [15]. FOXM1 is an anti-tumor target due to its role in tumorigenesis. Similarly, inhibition of CD44 function blocks tumor invasion and metastatic colonization [27]. Vimentin downregulation inhibits the invasion of breast cancer cells [28, 29]. Our results demonstrate that the anticancer effects of NCE may partially be attributable to the inhibition of CD31, FOXM1, CD44 and vimentin.

In conclusion, this is the first report to reveal the antiHCC effects of NCE from the supernatants of B. subtilis. NK treatment could increase survival and inhibit tumor growth in HCC mice, highlighting its potential as a novel anti-HCC therapeutic.

\section{Acknowledgments}

This work was funded by the National Natural Science Foundation of China (Grant no. 81702439, 81670549), Innovation Project for College Students of Jiangsu Universit (Grant no. 201810299009Z, 201810299100W), Young Backbone Teacher Training Project of Jiangsu University.

\section{Conflict of Interest}

The authors have no financial conflicts of interest to declare.

\section{References}

1. Nault JC, Galle PR, Marquardt JU. 2018. The role of molecular enrichment on future therapies in hepatocellular carcinoma. J. Hepatol. 69: 237-247.

2. Waziry R, Grebely J, Amin J, Alavi M, Hajarizadeh B, George J, et al. 2017. Survival following hospitalization with hepatocellular carcinoma among people notified with hepatitis B or C virus in Australia (2000-2014). Hepatol. Commun. 1: 736-47.

3. Najjar M, Agrawal S, Emond JC, Halazun KJ. 2018. Pretreatment neutrophil-lymphocyte ratio: useful prognostic biomarker in hepatocellular carcinoma. J. Hepatocell. Carcinoma 5: $17-28$.

4. Giard JM, Mehta N, Dodge JL, Roberts JP, Yao FY. 2018. Alpha-fetoprotein Slope $>7.5 \mathrm{ng} / \mathrm{ml} /$ month predicts micro- vascular invasion and tumor recurrence after liver transplantation for hepatocellular carcinoma. Transplantation 102: 816-822.

5. Weng Y, Yao J, Sparks S, Wang KY. 2017. Nattokinase: An oral antithrombotic agent for the prevention of cardiovascular disease. Int. J. Mol. Sci. 18(3). pii: E523.

6. Selvarajan E, Bhatnagar N. 2017. Nattokinase: an updated critical review on challenges and perspectives. Cardiovasc. Hematol. Agents Med. Chem. doi: 10.2174/1871525716666171207153332. [Epub ahead of print]

7. Kotb E. 2014. The biotechnological potential of fibrinolytic enzymes in the dissolution of endogenous blood thrombi. Biotechnol. Prog. 30: 656-672.

8. Fujita M, Ohnishi K, Takaoka S, Ogasawara K, Fukuyama R, Nakamuta H. 2011. Antihypertensive effects of continuous oral administration of nattokinase and its fragments in spontaneously hypertensive rats. Biol. Pharm. Bull. 34: 16961701.

9. Kim JY, Gum SN, Paik JK, Lim HH, Kim KC, Ogasawara K, et al. 2008. Effects of nattokinase on blood pressure: a randomized, controlled trial. Hypertens. Res. 31: 1583-1588.

10. Takano A, Hirata A, Ogasawara K, Sagara N, Inomata Y, Kawaji T, et al. 2006. Posterior vitreous detachment induced by nattokinase (subtilisin NAT): a novel enzyme for pharmacologic vitreolysis. Invest. Ophthalmol. Vis. Sci. 47: 2075-2079.

11. Fadl NN, Ahmed HH, Booles HF, Sayed AH. 2013. Serrapeptase and nattokinase intervention for relieving Alzheimer's disease pathophysiology in rat model. Hum. Exp. Toxicol. 32: 721-735.

12. Chand V, Pandey A, Kopanja D, Guzman G, Raychaudhuri P. 2019. Opposing Roles of the Fork-head box genes FoxM1 and FoxA2 in Liver Cancer. Mol. Cancer Res. 17: 1063-1074.

13. Guo D, Song X, Guo T, Gu S, Chang X, Su T, et al. 2018. Vimentin acetylation is involved in SIRT5-mediated hepatocellular carcinoma migration. Am. J. Cancer Res. 8: 2453-2466.

14. Wada F, Koga H, Akiba J, Niizeki T, Iwamoto H, Ikezono Y, et al. 2018. High expression of CD44v9 and $\mathrm{xCT}$ in chemoresistant hepatocellular carcinoma: potential targets by sulfasalazine. Cancer Sci. 109: 2801-2810.

15. Milewski D, Balli D, Ustiyan V, Le T, Dienemann H, Warth A, et al. 2017. FOXM1 activates AGR2 and causes progression of lung adenomas into invasive mucinous adenocarcinomas. PLoS Genet. 13: e1007097.

16. Zhang Bin, Liu Yuwan, Ji Qian, Zhao Mengmeng, Zeng Jian, Liu Lihua et al. 2017. Nattokinase Crude Extract Enhances Cutaneous Wound Healing. J. Biomater. Tissue Eng. 7: 1281-1286.

17. Man LL, Xiang DJ, Zhang CL. 2019. Strain screening from traditional fermented soybean foods and induction of Nattokinase production in Bacillus subtilis MX-6. Probiotics Antimicrob. Proteins 11: 283-294. 
18. Yin Y, Liu L, Zhao Z, Yin L, Bauer N, Nwaeburu CC, et al. 2018. Simvastatin inhibits sonic hedgehog signaling and stemness features of pancreatic cancer. Cancer Lett. 426: 14-24.

19. Ma C, Wang F, Han B, Zhong X, Si F, Ye J, et al. 2018. SALL1 functions as a tumor suppressor in breast cancer by regulating cancer cell senescence and metastasis through the NuRD complex. Mol. Cancer 17: 78.

20. Chatterji P, Rustgi AK. 2018. RNA Binding proteins in intestinal epithelial biology and colorectal cancer. Trends Mol. Med. 24: 490-506.

21. Incio J, Ligibel JA, McManus DT, Suboj P, Jung K, Kawaguchi K, et al. 2018. Obesity promotes resistance to anti-VEGF therapy in breast cancer by up-regulating IL-6 and potentially FGF-2. Sci. Transl. Med. 10(432).

22. Vaahtomeri K, Karaman S, Makinen T, Alitalo K. 2017. Lymphangiogenesis guidance by paracrine and pericellular factors. Genes Dev. 31: 1615-1634.

23. Murgai M, Ju W, Eason M, Kline J, Beury DW, Kaczanowska S, et al. 2017. KLF4-dependent perivascular cell plasticity mediates pre-metastatic niche formation and metastasis. Nat. Med. 23: 1176-90.

24. Suzuki Y, Kondo K, Matsumoto Y, Zhao BQ, Otsuguro K, Maeda T, et al. 2003. Dietary supplementation of fermented soybean, natto, suppresses intimal thickening and modulates the lysis of mural thrombi after endothelial injury in rat femoral artery. Life Sci. 73: 1289-1298.

25. Yao S, Fan LY, Lam EW. 2017. The FOXO3-FOXM1 axis: A key cancer drug target and a modulator of cancer drug resistance. Semin. Cancer Biol. 50: 77-89.

26. Yue M, Li S, Yan G, Li C, Kang Z. 2018. Paeoniflorin inhibits cell growth and induces cell cycle arrest through inhibition of FoxM1 in colorectal cancer cells. Cell Cycle 17: 240-249.

27. Zaiden M, Feinshtein V, David A. 2017. Inhibition of CD44v3 and CD44v6 function blocks tumor invasion and metastatic colonization. J. Control. Release 257: 10-20.

28. Nodale C, Sheffer M, Jacob-Hirsch J, Folgiero V, Falcioni R, Aiello A, et al. 2012. HIPK2 downregulates vimentin and inhibits breast cancer cell invasion. Cancer Biol. Ther. 13: 198205.

29. Zhang X, Fournier MV, Ware JL, Bissell MJ, Yacoub A, Zehner ZE. 2009. Inhibition of vimentin or beta1 integrin reverts morphology of prostate tumor cells grown in laminin-rich extracellular matrix gels and reduces tumor growth in vivo. Mol. Cancer Ther. 8: 499-508. 